\title{
Verification of He's frequency formulation by Duffing-Harmonic Oscillator
}

\author{
Z.L. Tao ${ }^{1}$ \\ ${ }^{1}$ Nanjing Univ Informat Sci \& Technol
}

June 28, 2020

\begin{abstract}
He's frequency formulation suggests the fast identification of the amplitude-frequency relationship of a nonlinear oscillator. This paper applies the formulation to the Duffing-harmonic oscillator with great success. A modification of He's frequency formulation and the variational iteration method are also used for comparison.
\end{abstract}

\section{A modification of He's frequency-amplitude formulation}

He's original frequency formulation was derived from an ancient Chinese mathematical algorithm [10, 13], recently there was a hot discussion on He's original frequency formulation [14-22]. Here we check Ren-Hu's modification [14].

Rewrite the problem (1) in this form

$\ddot{x}\left(1+x^{2}\right)+x^{3}=0, x(0)=A, \dot{x}(0)=0$.

In view of He's frequency-amplitude formulation [7-9], we use a trial solution

$x_{1}=A \cos \omega_{1} t$. (11)

Taking equation (11) into the first equation of problem (10) results in the residual

$R_{1}=-\omega_{1}^{2} A \cos \omega_{1} t\left(1+A^{2} \cos ^{2} \omega_{1} t\right)+A^{3} \omega_{1} t$

$=\left[\frac{3 A^{3}}{4}\left(1-\omega_{1}^{2}\right)-\omega_{1}^{2} A\right] \cos \omega_{1} t+\frac{A^{3}}{4}\left(1-\omega_{1}^{2}\right) \cos 3 \omega_{1} t$.

Introducing defined in [14]

$\tilde{R}_{1}=\frac{4}{T} \int_{0}^{\frac{T}{4}} R_{1}(t) \mathrm{dt},(13)$

with $T$ the period of the oscillator.

Submitting equation (12) into equation (13), we obtain

$\tilde{R}_{1}=\frac{4}{T} \int_{0}^{\frac{T}{4}}\left\{\left[\frac{3 A^{3}}{4}\left(1-\omega_{1}^{2}\right)-\omega_{1}^{2} A\right] \cos \omega_{1} t+\frac{A^{3}}{4}\left(1-\omega_{1}^{2}\right) \cos 3 \omega_{1} t\right\} \mathrm{dt}$

$=\frac{2}{\pi}\left[\frac{3 A^{3}}{4}\left(1-\omega_{1}^{2}\right)-\omega_{1}^{2} A\right]-\frac{\left(1-\omega_{1}^{2}\right) A^{3}}{6 \pi}(14)$

Let $\mathrm{x}_{2}=A \cos \omega_{2} t$ with $\omega_{1} \neq \omega_{2}$, by the similar operation as the above, we have

$\tilde{R}_{2}=\frac{2}{\pi}\left[\frac{3 A^{3}}{4}\left(1-\omega_{2}^{2}\right)-\omega_{2}^{2} A\right]-\frac{\left(1-\omega_{2}^{2}\right) A^{3}}{6 \pi} \cdot(15)$

Using He's frequency-amplitude formulation [10, 13], we have 
$\omega^{2}=\frac{\tilde{R}_{2} \omega_{1}^{2}-\tilde{R}_{1} \omega_{2}^{2}}{\tilde{R}_{2}-\tilde{R}_{1}}=\frac{\left\{\frac{2}{\pi}\left[\frac{3 A^{3}}{4}\left(1-\omega_{2}^{2}\right)-\omega_{2}^{2} A\right]-\frac{\left(1-\omega_{2}^{2}\right) A^{3}}{6 \pi}\right\}^{2}{ }^{2}-\left\{\frac{2}{\pi}\left[\frac{3 A^{3}}{4}\left(1-\omega_{1}^{2}\right)-\omega_{1}^{2} A\right]-\frac{\left(1-\omega_{1}^{2}\right) A^{3}}{6 \pi}\right\}^{2}{ }_{2}}{\frac{2}{\pi}\left[\frac{3 A^{3}}{4}\left(1-\omega_{2}^{2}\right)-\omega_{2}^{2} A\right]-\frac{\left(1-\omega_{2}^{2}\right) A^{3}}{6 \pi}-\left\{\frac{2}{\pi}\left[\frac{3 A^{3}}{4}\left(1-\omega_{1}^{2}\right)-\omega_{1}^{2} A\right]-\frac{\left(1-\omega_{1}^{2}\right) A^{3}}{6 \pi}\right\}}=\frac{18 A^{2}}{(9 \pi-2) A^{2}+24}$

When , Eq. (16) becomes

$\omega^{2}=\frac{18}{(9 \pi-2)}(17)$

while the exact one is $\omega=1$. When $\mathrm{A}<<1$, we have

$\omega^{2}=\frac{18 A^{2}}{24}=\frac{3 A^{2}}{4}$

This agrees with that by the homotopy perturbation method [10-12]. So Ren-Hu's modification is valid for $\mathrm{A}<<1$.

\section{Variational iteration method}

Reconsider the problem (1), according to the variational iteration method with Laplace transform; we have the iteration equality [23]

$L\left[x_{n+1}\right]=L\left[x_{n}\right]-L\left[\int_{0}^{t} \frac{1}{\omega} \sin \omega(t-\xi) \delta \xi\right]$

$=L\left[x_{n}\right]-\frac{1}{\omega} L[\sin \omega \tau] \bullet L\left[\ddot{x}_{n}(t)+x_{n}^{3}(t)+x_{n}^{2}(t) \ddot{x}_{n}(t)\right](19)$

where $\mathrm{L}$ is the Laplace transform operator.

Assume the initial approximation be

$x_{0}=A \cos \omega \tau(20)$

Then we have

$L\left[x_{1}(t)\right]=L[A \cos \omega \tau]-\frac{1}{\omega} L[\sin \omega \tau] \bullet L\left[-A \omega^{2} \cos \omega t+A^{3} \cos ^{3} \omega t-A^{3} \omega^{2} \cos ^{3} \omega \tau\right]$

$=L[A \cos \omega \tau]-\frac{1}{\omega} L[\sin \omega \tau] L\left[\left(\frac{3\left(A^{3}-A^{3} \omega^{2}\right)}{4}-A \omega^{2}\right) \cos \omega t+\frac{A^{3}-A^{3} \omega^{2}}{4} \cos 3 \omega \tau\right](21)$

Imposing the inverse Laplace transform on equality (21), there holds the first-order approximant

$x_{1}(t)=A \cos \omega \tau-\frac{t}{4 \omega}\left(3\left(A^{3}-A^{3} \omega^{2}\right)-4 A \omega^{2}\right) \sin \omega \tau+\frac{A^{3} \omega^{2}-A^{3}}{32 \omega^{2}}(\cos \omega \tau-\cos 3 \omega \tau)(22)$

No secular term in the equality (22) requires that

$3\left(A^{3}-A^{3} \omega^{2}\right)-4 A \omega^{2}=0(23)$

which yields this

$\omega^{2}=\frac{3 A^{2}}{4+3 A^{2}}$

Eq. (24) satisfies two scale extremals.

Discussion and Conclusions

Eq. (1) can be solved effectively by the homotopy perturbation method [24-28] and the variational approach $[2,29]$. By the semi-inverse method [30, 31], we can establish a variational formulation for Eq. (1), which is

$J(u)=\int_{0}^{T / 4}\left\{\frac{1}{2} \dot{x}^{2}-\frac{1}{2} x^{2}+\frac{1}{2} \ln \left(1+x^{2}\right)\right\} \operatorname{dt}(25)$

By comparison also with those in open literatures, we conclude that He's frequency is the simplest while its accuracy is also extremely high. Considering the simplest calculation, He's frequency formulation greatly promote the development of the nonlinear science, especially the nonlinear vibration. 


\section{References}

1. He, J-H., The simplest approach to nonlinear oscillators. Results in Physics, 2019; 15: 102546.

2. He JH. The simpler, the better: Analytical methods for nonlinear oscillators and fractional oscillators. Journal of Low Frequency Noise, Vibration and Active Control, 2019; 38(3-4): 1252-1260.

3. J.H. He, F.Y. Ji. Taylor series solution for Lane-Emden equation. Journal of Mathematical Chemistry, 2019; 57(8): 1932-1934.

4. H.Hu. Solution of a Duffing-harmonic oscillator by an iteration procedure. J. Sound Vibration, 2006; 298: 446-452.

5. H.Hu, J.H.Tang. Solution of a Duffing-harmonic oscillator by the method of harmonic balance. J. Sound Vibration, 2006; 298: 637-639.

6. Zhao-ling Tao. Frequency-amplitude relationship of the Duffing-harmonic oscillator. Topological methods in nonlinear analysis, 2008; 31: 279-285.

7. Beléndez A, A. Hernández, T. Beléndez, E. Fernández, M. L. Álvarez and C. Neipp. Application of He's Homotopy Perturbation Method to the Duffing-Harmonic Oscillator. International Journal of Non-linear Sciences and Numerical Simulation, 2007; 8: 79-88.

8. J.H. He, F.Y. Ji. Two-scale mathematics and fractional calculus for thermodynamics. Thermal Science, 2019; 23 (4): 2131-2133.

9. Ain, Q.T., He, J.H. On two-scale dimension and its applications. Thermal Science, 2019; 23(3B): 17071712.

10. J.H.He. Some asymptotic methods for strongly nonlinear equations. Int. J. Mod. Phys. B, 2006; 20 (10): 1141-1199.

11. He, J.H. Homotopy Perturbation Method with an Auxiliary Term. Abstract and Applied Analysis, 2012: 857612 .

12. He, J.H. Homotopy perturbation method with two expanding parameters. Indian Journal of Physics, 2014; 88: 193-196.

13. J.H.He An improved Amplitude-frequency formulation for nonlinear oscillators. International journal of nonlinear sciences and numerical simulation, 2008; 9(2): 211-212.

14. Zhong-fu Ren, Gui-fang Hu. He's frequency-amplitude formulation with average residuals for nonlinear oscillators. Journal of Low Frequency Noise, Vibration and Active Control, 2019; 38(3-4): 1050-1059.

15. Zhong-Fu Ren, Jin-Bin Wu. He's frequency-amplitude formulation for nonlinear oscillator with damping. Journal of Low Frequency Noise, Vibration and Active Control, 2019; 38(3-4):1045-1049.

16. Qingli Wang, Xiangyang Shi, Zhengbiao Li. A short remark on Ren-Hu's modification of He's frequency-amplitude formulation and the temperature oscillation in a polar bear hair. Journal of Low Frequency Noise, Vibration and Active Control, 2019; 38(3-4):1374-1377.

17. Zhong-Fu Ren, Gui-Fang Hu. Discussion on the accuracies of He's frequency-amplitude formulation and its modification with average residuals. Journal of Low Frequency Noise, Vibration and Active Control, 2019; 38(3-4):1713-1715.

18. Zhao-Ling Tao, Guo-Hua Chen, Kai Xian Bai. Approximate frequency-amplitude relationship for a singular oscillator. Journal of Low Frequency Noise, Vibration and Active Control, 2019; 38(3-4):10361040 .

19. Gui-Fang Hu, Shu-Xian Deng. Ren's frequency-amplitude formulation for nonlinear oscillators. Journal of Low Frequency Noise, Vibration and Active Control, 2019; 38(3-4):1681-1686.

20. Chun-Hui He, Jian-Hong Wang, Shao-wen Yao. A complement to period/frequency estimation of a nonlinear oscillator. Journal of Low Frequency Noise, Vibration and Active Control, 2019; 38(3-4): 992-995.

21. Dan Tian, Zhi Liu. Period/frequency estimation of a nonlinear oscillator. Journal of Low Frequency Noise, Vibration and Active Control, 2019; 38(3-4): 1629-1634.

22. Zhao-Ling Tao, Guo-Hua Chen, Yan-Mei Xue. Frequency and solution of an oscillator with a damping. Journal of Low Frequency Noise, Vibration and Active Control, 2019; 38(3-4): 1699-1702.

23. Naveed Anjum, He JH. Laplace transform: Making the variational iteration method easier, Applied Mathematics Letters, 2019, 92(1): 134-138 
24. Xiao-Xia Li, Chun-Hui He. Homotopy perturbation method coupled with the enhanced perturbation method. Journal of Low Frequency Noise, Vibration and Active Control, 2019; 38(3-4):1399-1403.

25. Dan-Ni Yu, Ji-Huan He, Andres G Garcıa. Homotopy perturbation method with an auxiliary parameter for nonlinear oscillators. Journal of Low Frequency Noise, Vibration and Active Control, 2019; 38(34):1540-1554.

26. Adamu, M.Y., Ogenyi, P. New approach to parameterized homotopy perturbation method. Thermal Science, 2018; 22(4): 1865-1870.

27. Liu, Z.J., Adamu, M.Y., Suleiman, E. Hybridization of homotopy perturbation method and Laplace transformation for the partial differential equations. Thermal Science, 2017; 21: 1843-1846.

28. Wu Y., He, J.H. Homotopy perturbation method for nonlinear oscillators with coordinate dependent mass. Results in Physics, 2018; 10: 270-271.

29. Yasir Nawaz, Muhammad Shoaib Arif, Mairaj Bibi, Mehvish Naz, Rabia Fayyaz. An effective modification of He's variational approach to a nonlinear oscillator. Journal of Low Frequency Noise, Vibration and Active Control, 2019; 38(3-4):1013-1022.

30. J.H. He. A modified Li-He's variational principle for plasma, International Journal of Numerical Methods for Heat and Fluid Flow, (2019) DOI: 10.1108/HFF-06-2019-0523

31. J.H. He, Lagrange Crisis and Generalized Variational Principle for 3D unsteady flow, International Journal of Numerical Methods for Heat and Fluid Flow, 2020; 30(3):1189-1196. 